\title{
The impact of false-positive newborn screening results on families: a qualitative study
}

\author{
Johanna L. Schmidt, MPH, MGC1, Karen Castellanos-Brown, PhD², Saltanat Childress, MSW³, \\ Natasha Bonhomme, BA ${ }^{4}$, Julianne S. Oktay, PhD ${ }^{3}$, Sharon F. Terry, MA ${ }^{4}$, Penny Kyler, OT, ScD ${ }^{5}$, \\ Amy Davidoff, $\mathrm{PhD}^{6}$ and Carol Greene, $\mathrm{MD}^{7}$
}

Purpose: Newborn screening leads to improved treatment and disease outcomes, but false-positive newborn screening results may cause distress for parents. The purpose of this study was to describe the experiences of families who receive a false-positive newborn screening result in an attempt to discover ways to help improve the newborn screening communication process for families.

Methods: This was a qualitative study using two methods of data collection: in-depth, semistructured interviews and focus groups. Participants $(N=27)$ were parents whose children (ages 6-16 months) underwent follow-up testing after newborn screening and whose follow-up test results indicated that the newborn screening result was a false-positive.

Results: Our analysis found that parents who have a false-positive newborn screening result experience five distinct stages. Most par-

\section{INTRODUCTION}

Newborn screening (NBS), widely regarded as a successful public health initiative, is mandated throughout the United States. Although NBS leads to improved treatment and disease outcomes, there remain some challenges, including the scenario in which an infant is identified as needing additional testing based on an out-of-range screening result, followed by diagnostic testing that indicates the infant is unaffected. This is called a false-positive (FP) NBS.

The exact FP rate varies according to screening method, condition, and population. ${ }^{1}$ One study ${ }^{2}$ estimates a minimum FP rate of $0.33 \%$ or $1 / 300$ infants. If correct, then, with the US birth rate of four million a year, ${ }^{3}$ more than 13,000 families each year may experience FP NBS, and this may be a low estimate. Previous research suggests that parents experience stress and anxiety when their child is retested after a positive NBS and negative effects may persist even when the result is an FP. ${ }^{4-7}$ This study uses qualitative methods to provide a detailed description of the experiences of families in three US states who had an FP NBS.

\section{MATERIALS AND METHODS}

\section{Design}

We present results of the qualitative component of a consecutive mixed-methods study, a model in which a qualitative ents did not report long-term negative impacts of the experience, but some experienced some residual worry. Participants described effective provider communication as key in mitigating stress. Some parents identified the experience as leading to positive outcomes.

Conclusion: Identifying best practices for communication between the health care providers and parents is an essential component in improving the newborn screening process. Further research is needed to discover best practices for communication to minimize potential harm and maximize the benefits of newborn screening.

Genet Med 2012:14(1):76-80

Key Words: communication; false-positive; newborn screening; parental stress; qualitative

component is conducted prior to a quantitative component. ${ }^{8}$ Qualitative research is appropriate for our purposes because the experience of FP NBS is not well understood and because our aim is to describe a process. ${ }^{9}$ The qualitative study had two parts: in-depth, semistructured interviews and focus groups.

The study was designed to capture the experiences of families who experienced FP NBS. Because this is a qualitative study of experience, we did not study normal controls. We did study, as positive controls, parents of children with a heart murmur at birth who proved to have no heart disease after a cardiology evaluation.

\section{Sample}

We used a convenience sample made up of parents who met our eligibility criteria and who were recruited with the help of state health departments in three US states: Maryland, Michigan, and Georgia. Eligible parents had a child older than 6 months but younger than 18 months who had follow-up testing after positive NBS for any condition and whose follow-up test results indicated that the NBS result was an FP. Parents were ineligible if the child had serious medical problems at birth or if parents were less than 18 years old or did not speak fluent English. Two heart murmur positive controls were included. 


\section{Procedures}

Participants were recruited by the genetics clinic and the various state NBS coordinators. Consent was obtained by the study coordinator. Incentives were used to recruit. Approval from the institutional review boards of the University of Maryland, the Maryland Department of Health and Mental Hygiene, the Michigan Department of Community Health, Emory University, and the Georgia Division of Public Health were obtained and maintained.

\section{Data gathering}

Semistructured interviews were conducted from November 2008 until June 2009. Interviews lasted between 15 and $75 \mathrm{~min}$. In-person interviews were impractical for most respondents, who preferred telephone interviews. Two interviews were completed in person, and the remainder were done by phone. Two focus groups were completed: one in Michigan in September 2009 and one in Georgia in November 2009. Using concepts and themes from initial analysis of interviews, an interview guide for the focus groups was created.

\section{Data analysis}

Interviews and focus groups were audio-recorded, transcribed verbatim, and analyzed using NVivo7 qualitative data analysis software. We used grounded theory methods to code interviews. ${ }^{10-12}$ We coded completed interviews as new interviews were being conducted and used emerging concepts to guide further data gathering. We began with open coding, identifying "in vivo" codes as well as conceptual codes. ${ }^{10-12}$ The coding scheme developed from interviews was adapted as focus group data were analyzed. We used iterative processes to validate concepts. Three members of the team, representing two disciplines, coded transcripts and met periodically to resolve discrepancies. To protect privacy, in this article, we identified participants by a pseudonym. To facilitate presentation, we used qualitative terms "some," "many," and, "most"; "some" means more than two, but less than half; "many" means more than half; and "most" means all but two or three.

\section{Participant characteristics}

\section{RESULTS}

Sixteen interviews were completed $(N=16)$, which included 14 mothers, one couple, and one father. Five mothers participated in the Michigan focus group $(N=5)$ and a mother and two couples participated in the Georgia focus group $(N=5)$. The infants had screened positive for various conditions, including congenital hypothyroidism, congenital adrenal hyperplasia (OMIM\# 201910), galactosemia (OMIM\# 230400), biotinidase deficiency (OMIM\# 253260), cystic fibrosis (OMIM\# 219700), and conditions detected by tandem mass spectrometry (see Table 1). The infants were 6-16 months of age.

Parents interviewed were between 22 and 42 years of age with a median age of 34 years. Almost all were married. Their educational levels ranged from high school to postgraduate, with "completed 4 years of college" the most common category.
Table 1 Semistructured interviews

\begin{tabular}{|c|c|}
\hline Type of interview & $\begin{array}{c}\text { Number } \\
\text { of interviews }\end{array}$ \\
\hline False-positives & 14 \\
\hline Tandem mass spectrometry & 7 \\
\hline $\begin{array}{l}\text { Endocrinopathies (e.g., congenital adrenal } \\
\text { hyperplasia) }\end{array}$ & 2 \\
\hline $\begin{array}{l}\text { Enzyme deficiencies (e.g., galactosemia, } \\
\text { biotinidase deficiency) }\end{array}$ & 2 \\
\hline Cystic fibrosis (elevated immunoreactive trypsinogen) & 3 \\
\hline Controls & 2 \\
\hline Heart murmur evaluation & 2 \\
\hline Total & 16 \\
\hline
\end{tabular}

About half worked outside of the home (or were students) and about half were stay-at-home mothers. Three of the mothers said that they were on Medicaid, a state health insurance program. We did not collect specific demographic information from focus group participants, but they appeared to have a similar profile to the interviewees.

Two families with children with short-term health problems were inadvertently interviewed because this information was not discovered until well into the interview. We included these cases in the analysis when we discovered that their experiences did not differ from those of other parents with FP newborn screens.

\section{The FP experience}

By analysis of transcripts, field notes, and participant observation, we found that parents who have an FP NBS experience five distinct stages: (i) receiving the news, (ii) getting the follow-up testing, (iii) waiting for resolution, (iv) learning the follow-up test results, and (v) adapting to life after NBS. For each stage, we describe the process and parents' emotional responses and coping.

\section{Receiving the news}

Because the initial positive NBS result represents a threat to the baby's health, all parents experienced stress after hearing the news, ranging from mild concern to severe anxiety. One mother (Helen) said, "I'm starting to get emotional here, but it was very stressful for me ... I was thinking, 'Oh my gosh, is he going to die?"”

Most parents were told of the positive NBS by their child's pediatrician, by phone or in person at their next scheduled visit. Parents appreciated contact with their child's pediatrician because their child's pediatrician provided reassurance. One of the parents (Carol) said, "She is great. She told us not to worry. She said, "It's probably going to be a false positive." She was explaining that in all her years that ... she only had 2 cases where it ever was positive."

Parents notified by a person not known to them, such as someone from the State Health Department, tended to be dissatisfied, as were parents who felt their pediatricians did not fully answer 
their questions. Some parents seemed to experience more anxiety after receiving information on days before a weekend or holiday and not being able to follow up immediately.

\section{Getting follow-up testing}

Different follow-up tests were performed depending on the disorder. Some had to go to a special facility, whereas others could bring the child to their regular pediatrician. The retesting procedure was sometimes stressful for parents. One mother (Katelyn) described her experience, "So, when she pricked my baby's feet ... it looked so painful ... he started to cry, and it's like, 'Oh my goodness, I'm just torturing my child, just for this.'”

Some parents were upset when the child had to go through multiple retesting procedures. Yolanda, whose child had to be tested five times, said, "The state kept on saying, you know, 'Okay, his levels were low, but they weren't low enough, come back.' So much that when [my baby] saw the nurses, he would just cry."

In response to questions about financial burden, parents did not report concern about cost of follow-up testing. Nearly all parents in this study reported having insurance, which probably accounts for their not having to pay high costs for retesting. Most had a co-pay but expressed that it was not a problem. Although many did experience one or more indirect costs (e.g., missed work, time, and gas), most were not upset about this.

\section{Waiting for resolution}

For most parents, the anxiety experienced upon learning the initial results of the NBS continued until they learned the result was an FP. One mother, Margaret, described the period as "a nightmarish two and a half weeks."

Elizabeth, another mother, described how nothing could calm her worry: "Nothing made me feel better ... If God had come down, if a prophet had come down from heaven and told me that he did not have cystic fibrosis, I would not have believed it until I had got the sweat test."

To cope with anxiety during the waiting period, parents relied on two coping mechanisms: seeking information and seeking social support.

Seeking information. Seeking information is a way of increasing control by learning what to expect and how to prevent unwanted consequences. ${ }^{13,14}$ Many parents turned to the Internet for information. Some reported feeling reassured by information they found. One mother (Shirley) was reassured because her child did not have the symptoms that she had read were associated with the disease in question: "I was paying closer attention to her, making sure she doesn't have any of these three symptoms: the vomiting, lethargic [sic], smell."

Other parents, in particular parents of children who had an FP cystic fibrosis screen, became more anxious when they read about the condition, alarmed by words such as "terminal."

Some parents chose not to seek online information, as a kind of self-protection. A father, Robert, explained, "If I went online and tried to figure out what this was all about and all the potential health problems and risks, I wouldn't have been able to live a normal life."

Seeking social support. Although seeking social support can be an important coping mechanism for handling stressful situations ${ }^{15}$ this was complicated for many parents with a positive NBS. To seek support, parents would need to share information about the NBS results. Parents went through an appraisal process in deciding with whom to share the information, weighing costs and potential benefits. Some parents chose not to share the information about the NBS as a type of self-protection based on their assessment of how others would react. One mother (Carol) explained, "I felt that if less people knew it would be easier on me ... I decided that in order to avoid people volunteering information that I would just not involve them until I knew for sure that it was something that they needed to know."

Some mothers chose to share information about the initial positive result only with their spouse or a few close people to protect others from unnecessary worry. Samantha said, "Why panic everybody about something that, you know, that could either be or not be?"

For some, using social support was a positive experience. Louise described talking to her family: "They were trying to assure me that everything was probably fine and she seemed healthy and was eating well, and, you know, even if it was a problem it wasn't something that was really life threatening at that point."

Another mother, Kelly, said she appreciated that friends validated her feelings of sadness: "I told just a few very close to me people that could be supportive of me at that time and not tell me it's going to be okay. To tell me it's okay to be sad, and it's okay to feel how you feel."

Maintaining optimism. Not all parents experienced difficulty during the waiting period. For some, the initial reassurance by the pediatrician (or other trusted people) was adequate to minimize anxiety. Others simply trusted their own intuition. Still others used prayer and faith to cope. Some resolved to be confident, believing they could deal with whatever result came back from follow-up testing.

\section{Learning the follow-up test result}

Most parents learned that the NBS had been an FP through a phone call from their physician. However, in two cases, no notification of the follow-up test results was ever given and these parents assumed the follow-up test was normal. For most parents, anxiety dissipated the moment they heard that the test had been an FP. One mother (Robyn) said, "So, it was definitely a sigh of relief, of kinda, 'Ok, I can scratch that off my list and now focus on other stuff."'

Most participants quickly put the anxiety they felt going through the process behind them. They make the point that worry is a normal part of being a parent and that with or without NBS, they worry about their children. Joan explained, "I mean as far as the genetics and stuff like that, I don't have any more worries about that at all. I just have normal mother worries." 


\section{Adapting to life after NBS}

When asked about any lasting negative impact of the NBS experience, most said there was no long-term impact. However, some reported some lingering anxiety, sometimes triggered by symptoms related to the disease in question and sometimes by memories of the testing experience. About a third described a positive long-term impact of the experience with the FP NBS. For some, it helped them set priorities and focus on the important things. Some felt appreciation and gratitude for the experience or felt they gained perspective on the important things in life. Robert felt this was his first real challenge as a parent and was proud of how he handled it: "You know, I would say being my real, my true first test as a father, you know, it would have been very easy to crack up and fold under the pressure and panic ... I'm kind of proud of myself for keeping my wife together."

Another common positive outcome was in the relationship between parents and pediatrician. Francine said, "It just reassured me that I picked the right pediatrician because she was very thorough and I know if something was going on she would contact the people that needed to be contacted ... My children are going to a good doctor."

Some parents changed their opinion of a pediatrician that they had not liked before as a result of the NBS experience. One mother (Carla) said, "I feel like he's probably a better doctor than I thought because I didn't really think he would know much about it, but he seemed pretty knowledgeable about it."

\section{Suggestions for improvement in NBS}

We asked parents of babies with an FP NBS to suggest ways that their experiences could have been improved. There was a consensus among parents that health providers need to do a better job communicating about NBS. Suggestions included providing more information, including a clear explanation of NBS; telling parents what to expect, including how long they will have to wait for test results; providing information about the likelihood of an FP result; and providing information about the symptoms of the disease. Parents also mentioned wanting more help with their anxiety, more support, improved communication skills on the part of the health professional, and a need for health providers to show care and recognize their anxiety. In addition, parents suggested the need for increased efforts to shorten the waiting time, reduce the pain of the test (e.g., heel stick), and otherwise make the process easier on parents and babies alike.

\section{DISCUSSION}

We found that parents who receive an FP NBS go through a series of stages. The experience varies somewhat depending on the disease in question because the retesting procedures were very different. A major factor was the length of the waiting period. It seemed that the longer they had to wait for the results of the testing, the greater the anxiety. For example, for the heart murmur patients, the waiting time was limited to the time it took to get an appointment; the results of the retest were known the minute the cardiologist listened to the baby's heart. In contrast, the parents who needed a sweat test sometimes had to wait a long time for the procedure and the results. Parental needs for information and support vary depending on the stage. One of the most consistent findings was the importance of communication between parents and physicians.

Like parents in a recent study, ${ }^{6}$ some sought information from the Internet to relieve their anxiety, but this sometimes increased anxiety. In addition to seeking information, parents turned to family members (and others) for social support. However, many decided not to share the information about their baby's NBS result, either out of self-protection or to protect family members from anxiety. Social support was often limited to support from the other parent.

The stages we identified and coping strategies parents used were strikingly similar to those found in a qualitative study done with cystic fibrosis families. ${ }^{6}$ This serves to validate our findings and suggests that stages are consistent across various genetic conditions. Further qualitative studies on the experience of parents with FP NBS are needed to test and extend our finding of a staged experience with five distinct stages.

Our results suggest that parental anxiety occurs primarily between stages one and four. This anxiety for most parents decreased immediately upon learning that the test result was negative. This is consistent with other research. ${ }^{5,16,17}$ We also found that anxiety was reduced when parents were informed by their pediatricians and when he/she is able to provide immediate information and support. These results were consistent with research that suggests provider communication is a key factor in decreasing the emotional impact of learning the NBS result, in that those who provided understandable information could help mitigate distress. ${ }^{4-6,18}$

Because our study used qualitative methodology to describe the experience of parents who received FP NBS results, it was not designed to address the questions, raised in the literature, of whether FP NBS has a negative impact on the long-term relationship of the parent and child ${ }^{4-6,19-21}$ or whether the FP result affects how parents perceive their child's health., ${ }^{4,1921}$ Even so, in our study, most parents did not report any long-term impact on their relationship with their baby. Further quantitative research studies with large samples would be needed to answer these questions.

Our study also showed that many parents experienced positive impact such as growth in their parental role and strengthening of the relationship between spouses or between parents and physician. Overall, many parents described NBS as a necessary measure to prevent future disease.

\section{Implications}

Given these findings, emphasis should be placed on providing more accessible, understandable, and credible information on the NBS process and fostering better communication between health-care providers and parents, including better explanation of the NBS process in general and FP results in particular. Our results suggest that education of expectant and new parents about the likelihood (and meaning) of an FP result may help 
reduce anxiety. Also, efforts to improve providers' knowledge and communication skills may help them to do a better job in communicating with and assisting parents to better cope with the anxiety and stress when NBS results are not normal.

The FP experience can provide an opportunity for parents to gain confidence in their pediatricians and forge a stronger relationship. Pediatricians can help parents by contacting them directly regarding the news of an abnormal NBS and with followup news when the test is an FP. Because pediatricians and other primary care providers are central to the NBS process, attention needs to be focused on ensuring that they have the knowledge and communication skills necessary for handling abnormal screens in optimal ways. Research is needed to identify what works best. Training for providers in the stages that parents experience, their needs at each stage, and how to identify and support parents who are especially anxious is important. Further research is also needed to identify characteristics of parents who are likely to experience higher levels of anxiety and to identify procedures that will reduce or prevent adverse effects. Referrals to genetic counselors or social workers may help alleviate symptoms of lingering anxiety experienced by some parents.

\section{Limitations}

Because this was a small qualitative study, results cannot be generalized to all parents or even to a specific group of parents. Our results are based on an inductive strategy designed to develop theories, not to test them. The implications discussed are valid only to the extent that our results are supported in other studies. A quantitative study testing some hypotheses identified through this qualitative study is under way.

\section{Conclusion}

Because it is a screening test, FP results are inevitable in NBS. This study helps lay the foundation for identifying ways in which families are affected by FP NBS results. We identified areas in which improved education and resources may reduce any negative impact. More work is needed both to identify additional characteristics of parents who are likely to experience distress regarding receipt of FP results and to develop specific educational strategies and training for health providers in order to better support those parents. Also, continued education of the parent community regarding positive and FP results is needed. Using our results, it should be possible to develop strategies to minimize parent anxiety and maximize positive outcomes.

\section{ACKNOWLEDGMENTS}

This research was funded by the Health Resources and Services Administration grant U33MC07949, through a subcontract with the Genetic Alliance. Special thanks are due to Alex Agthe, Miriam Blitzer, Shannan Dixon, Antolin Llorente, and Susan Panny for assistance in development of study instruments and to Carolyn Dinsmore, Carrie Blout, Carole Flevaris, Janice Bach, Patricia Page, and
W. Andrew Faucett for their assistance in recruiting participants. We are very grateful to the families who participated in this study. They generously shared their experiences and suggestions, with the goal of helping to improve the NBS process for other families.

\section{DISCLOSURE}

The authors declare no conflict of interest.

\section{REFERENCES}

1. Tarini BA, Christakis DA, Welch HG. State newborn screening in the tandem mass spectrometry era: more tests, more false-positive results. Pediatrics 2006;118:448-456.

2. Schulze A, Lindner M, Kohlmüller D, Olgemöller K, Mayatepek E, Hoffmann GF. Expanded newborn screening for inborn errors of metabolism by electrospray ionization-tandem mass spectrometry: results, outcome, and implications. Pediatrics 2003;111(6 Pt 1):1399-1406.

3. Centers for Disease Control and Prevention, National Center for Health Statistics: Births, Marriages, Divorces and Deaths: Provisional Data for July 2009. National Vital Statistics Report 2009. <http://www.cdc.gov/nchs/data/nvsr/ nvsr58/nvsr58_15htm. Accessed 31 August 2010>.

4. Gurian EA, Kinnamon DD, Henry JJ, Waisbren SE. Expanded newborn screening for biochemical disorders: the effect of a false-positive result. Pediatrics 2006;117:1915-1921.

5. Sorenson JR, Levy HL, Mangione TW, Sepe SJ. Parental response to repeat testing of infants with 'false-positive' results in a newborn screening program. Pediatrics 1984;73:183-187.

6. Tluczek A, Koscik RL, Modaff P, et al. Newborn screening for cystic fibrosis: parents' preferences regarding counseling at the time of infants' sweat test. J Genet Couns 2006;15:277-291.

7. Morrison DR, Clayton EW. False positive newborn screening results are not always benign. Public Health Genomics 2011;14:173-177.

8. Creswell JW, Clark VLP. Designing and Conducting Mixed Methods Research SAGE: Thousand Oaks, CA, 2007.

9. Crabtree BF, Miller WL. Doing Qualitative Research, 2nd edn. SAGE: Thousand Oaks, CA, 1999

10. Charmaz K. Constructing Grounded Theory: A Practical Guide Through Qualitative Analysis. SAGE: Thousand Oaks, CA, 2006.

11. Corbin JM, Strauss AL. Basics of Qualitative Research, 3rd edn. SAGE: Thousand Oaks, CA, 2008

12. Glaser BSA. The Discovery of Grounded Theory. Aldine: Chicago, IL, 1967.

13. Carver C. Stress, coping \& health. In: Friedman HS, Silver RC (eds) Foundations of Health Psychology. Oxford University Press: New York, 2007: 117-144.

14. Sapolosky R. Why Zebras Don't Get Ulcers: An Updated Guide to StressRelated Diseases and Coping. Freeman: New York, 1998.

15. Taylor S. Social support. In: Friedman HS, Silver RC (eds). Foundations of Health Psychology. Oxford University Press: New York, 2007:145-171.

16. Bodegård G, Fyrö K, Larsson A. Psychological reactions in 102 families with a newborn who has a falsely positive screening test for congenital hypothyroidism. Acta Paediatr Scand Supp/ 1983;304:1-21.

17. Beucher J, Leray E, Deneuville E, et al. Psychological effects of false-positive results in cystic fibrosis newborn screening: a two-year follow-up. J Pediatr 2010;156:771-6, 776.e1

18. Davis TC, Humiston SG, Arnold CL, et al. Recommendations for effective newborn screening communication: results of focus groups with parents, providers, and experts. Pediatrics 2006;117(5 Pt 2):S326-S340.

19. Fyrö K, Bodegård G. Four-year follow-up of psychological reactions to false positive screening tests for congenital hypothyroidism. Acta Paediatr Scand 1987;76:107-114.

20. Waisbren SE, Albers S, Amato S, et al. Effect of expanded newborn screening for biochemical genetic disorders on child outcomes and parental stress. JAMA 2003;290:2564-2572.

21. Hewlett J, Waisbren SE. A review of the psychosocial effects of false-positive results on parents and current communication practices in newborn screening. $J$ Inherit Metab Dis 2006;29:677-682. 